\title{
THE CROSSING NUMBERS OF PRODUCTS WITH CYCLES
}

\author{
Emília DRAŽENSKÁ \\ Department of Mathematics and Theoretical Informatics, Faculty of Electrical Engineering and Informatics, Technical University of \\ Košice, Letná 9, 04200 Košice, Slovak Republic, tel.: +421 55602 2445, e-mail: emilia.drazenska@tuke.sk
}

\begin{abstract}
The crossing numbers of Cartesian products of all graphs of order at most four with cycles are known. The crossing numbers of Cartesian products $G \square C_{n}$ for several graphs $G$ on five and six vertices and the cycle $C_{n}$ are also given. In this paper, we extend these results by determining crossing numbers of Cartesian products $G \square C_{n}$ for some specific six vertex graphs $G$ and for some fixed number $n=3,4,5$.
\end{abstract}

Keywords: graph, Cartesian product, crossing number, cycle, drawing

\section{INTRODUCTION}

Let $G$ be a simple graph with vertex set $V$ and edge set $E$. A drawing of the graph in the plane is called a good drawing if and only if no edge crosses itself, no two edges cross more than once, and no two edges incident with the same vertex cross. The crossing number $\operatorname{cr}(G)$ of a graph $G$ is the minimum number of crossings of edges in a drawing of $G$ in the plane such that no three edges cross in a point. A drawing with minimum number of crossings is always a good drawing.

It is very difficult to establish the crossing number of a given graph. So, the crossing numbers are known only for a few families of graphs. Most of these graphs are Cartesian products of special graphs. The Cartesian product $G_{1} \square G_{2}$ of graphs $G_{1}$ and $G_{2}$ has vertex set $V\left(G_{1} \square G_{2}\right)=V\left(G_{1}\right) \square V\left(G_{2}\right)$ and edge set $E\left(G_{1} \square G_{2}\right)=$ $\left\{\left\{\left(u_{i}, v_{j}\right),\left(u_{k}, v_{h}\right)\right\}:\left(u_{i}=u_{k}\right.\right.$ and $\left.\left\{v_{j}, v_{h}\right\} \in E\left(G_{2}\right)\right)$ or $\left(\left\{u_{i}, u_{k}\right\} \in E\left(G_{1}\right)\right.$ and $\left.\left.v_{j}=v_{h}\right)\right\}$.

Let $C_{n}$ be the cycle of length $n, P_{n}$ be the path of length $n$, and $S_{n}$ be the star isomorphic to $K_{1, n}$. Harary et al. [9] conjectured that the crossing number of $C_{m} \square C_{n}$ is $(m-2) n$, for all $m, n$ satisfying $3 \leq m \leq n$. This has been proved only for $m, n$ satisfying $m \leq 7$ [1], [4], [17], [18], [19]. It was recently proved by Glebsky and Salazar [8] that the crossing number of $C_{m} \square C_{n}$ equals its long-conjectured value at least for $n \geq m(m+1)$. Beineke and Ringeisen in [2] as well as Jendrol' and Ščerbová in [10] determined the crossing numbers of the Cartesian products of all graphs on four vertices with cycles. Klešč in [11], [12], [13], [14], Klešč, Richter and Stobert in [15], and Klešč and Kocúrová in [16] gave the crossing numbers of $G \square C_{n}$ for several graphs $G$ of order five.

We are interested in the crossing numbers of Cartesian products of graphs on six vertices with cycles. Except for the star $S_{5}$, in [6] there are given the crossing numbers of $G \square C_{n}$ for all five-edge graphs $G$ on six vertices. In [7],the values of crossing numbers for sevetal Cartesian products of cycles and six-edge graphs $G$ on six vertices are presented. In [7] and [5] are given the crossing numbers for Cartesian products of cycles and two seven-edge graphs $G$ on six vertices. In this paper, we give the crossing number of the Cartesian products $G \square C_{n}$ for two graphs $G$ on six vertices and fixed number $n$.

\section{THE CROSSING NUMBERS OF $S_{5} \square C_{3}$ AND} $S_{5} \square C_{4}$

In [6] there is presented only upper bound $4 n$ for the crossing numbers of Cartesian products of star on six vertices with cycles $S_{5} \square C_{n}$ obtained from the drawing of the graph $S_{5} \square C_{n}$ for $n \geq 3$. We suppose that the upper bound in [6] is stated for $n \geq 6$. This bound is lower for $n=3,4,5$. In the next text we determine that $\operatorname{cr}\left(S_{5} \square C_{3}\right)=4$ and $\operatorname{cr}\left(S_{5} \square C_{4}\right)=8$. The hypothesis about lower bound for $n=5$, using the drawing of the graph $S_{5} \square C_{5}$, is 16 .

Theorem 2.1. $\operatorname{cr}\left(S_{5} \square C_{3}\right)=4, \operatorname{cr}\left(S_{5} \square C_{4}\right)=8$.

Proof. The graph $S_{5} \square C_{3}\left(S_{5} \square C_{4}\right)$ contains the graph $S_{5} \square P_{2} \quad\left(S_{5} \square P_{3}\right)$ as a subgraph. Bokal [3] proved that $\operatorname{cr}\left(S_{5} \square P_{n}\right)=4(n-1)$. Thus $\operatorname{cr}\left(S_{5} \square C_{3}\right) \geq 4$ $\left(\operatorname{cr}\left(S_{5} \square C_{4}\right) \geq 8\right)$. In Fig. 1 there are good drawings of $S_{5} \square C_{3}$ and $S_{5} \square C_{4}$ with four and eight crossings, respectively, therefore $\operatorname{cr}\left(S_{5} \square C_{3}\right) \leq 4$ and $\operatorname{cr}\left(S_{5} \square C_{4}\right) \leq 8$.

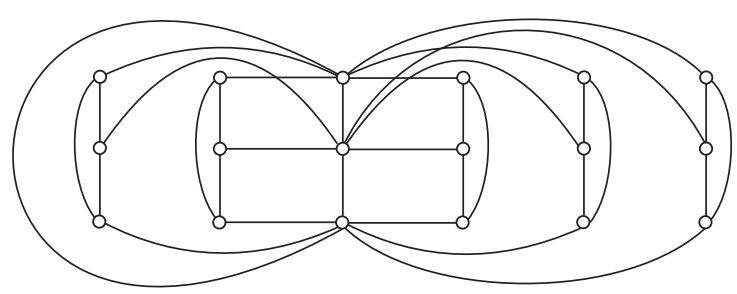

a)

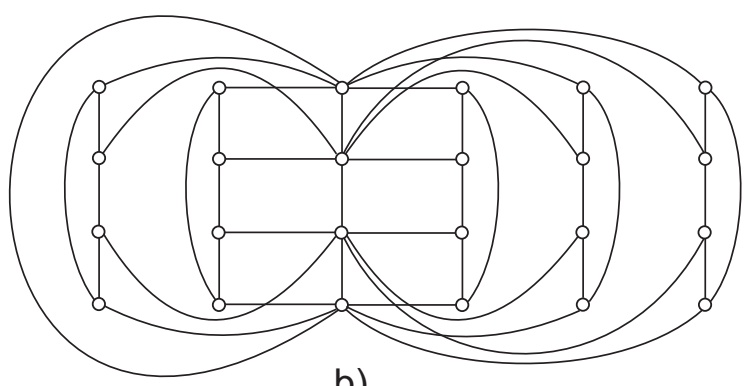

b)

Fig. 1 The drawings of the graphs $S_{5} \square C_{3}$ and $S_{5} \square C_{4}$ 
3. THE CROSSING NUMBERS OF $G \square C_{N}$ FOR THE SPECIFIC SIX-EDGE GRAPH $G$ AND FOR $N=$ $3,4,5$

For $n \geq 6$ - what is the upper boud? What about the exact value of the crossing number in this case?

At least - formulate the hypothesis.

In this section, we give the crossing numbers of the Cartesian products $G \square C_{3}, G \square C_{4}$ and $G \square C_{5}$ for the graph $G$ shown in Fig. 2. We prove, that $\operatorname{cr}\left(G \square C_{3}\right)=5$, $\operatorname{cr}\left(G \square C_{4}\right)=10$ and $\operatorname{cr}\left(G \square C_{5}\right)=14$. Fig. 3 shows the drawing of the graph $G \square C_{n}$ in which the edges of every subgraph isomorphic to $G$ are crossed exactly three times. Hence, the crossing number of $G \square C_{n}$ for $n \geq 6$ is at most $3 n$, we conjecture that it is exactly $3 n$.

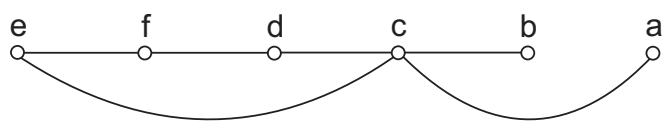

Fig. 2 The graph $G$

Let $D$ be a good drawing of the graph $G$. We denote the number of crossings in $D$ by $\operatorname{cr}_{D}(G)$. Let $G_{i}$ and $G_{j}$ be edge-disjoint subgraphs of $G$. We denote by $\operatorname{cr}_{D}\left(G_{i}, G_{j}\right)$ the number of crossings among edges of $G_{i}$ and edges of $G_{j}$, and by $\operatorname{cr}_{D}\left(G_{i}\right)$ the number of crossings between edges of $G_{i}$ in $D$.

Assume $n \geq 3$, and consider the graph $G \square C_{n}$ in the following way: it has $6 n$ vertices and edges that are the edges in the $n$ copies $G^{i}, i=0,1, \ldots, n-1$, and in the six cycles of length $n$. For $i=0,1, \ldots, n-1$, let $a_{i}$ and $b_{i}$ be the vertices of $G^{i}$ of degree one, $c_{i}$ the vertex of degree four and let $d_{i}$, $e_{i}$ and $f_{i}$ be the vertices of $G^{i}$ of degree two (see Fig. 3). Thus, for $x \in\{a, b, c, d, e, f\}$, the $n$-cycle $C_{n}^{x}$ is induced by the vertices $x_{0}, x_{1}, \ldots, x_{n-1}$.

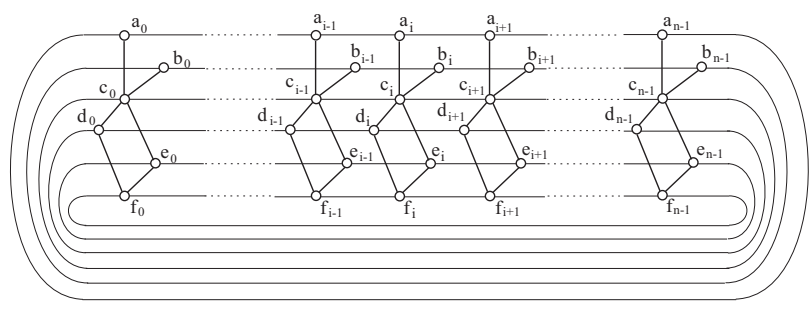

Fig. 3 The drawing of the graph $G \square C_{n}$

Let $T^{x}, \quad x \in\{a, b, d, e\}$, be the subgraph of the graph $G \square C_{n}$ consisting of the cycle $C_{n}^{x}$ together with the vertices of $C_{n}^{c}$ and of the edges joining $C_{n}^{x}$ with $C_{n}^{c}$. Let $X^{f}$ be the subgraph of $G \square C_{n}$ induced by the edges incident with the vertices of $C_{n}^{f}$. It is easy to see that $T^{a}, T^{b}, T^{d}, T^{e}, C_{n}^{c}$, and $X^{f}$ are edge-disjoint subgraphs and that

$$
G \square C_{n}=T^{a} \cup T^{b} \cup C_{n}^{c} \cup T^{d} \cup T^{e} \cup X^{f} .
$$

The subgraph $T^{a} \cup T^{b} \cup C_{n}^{c} \cup T^{d} \cup T^{e}$ of the graph $G \square C_{n}$ is isomorphic to the graph $S_{4} \square C_{n}$ and the subgraph $C_{n}^{c} \cup T^{d} \cup T^{e} \cup X^{f}$ of the graph $G \square C_{n}$ is isomorphic to the graph $C_{4} \square C_{n}$.
Theorem 3.1. $\operatorname{cr}\left(G \square C_{3}\right)=5$.

Proof. Fig. 4 shows the good drawing of the graph $G \square C_{3}$ with five crossings, thus $\operatorname{cr}\left(G \square C_{3}\right) \leq 5$.

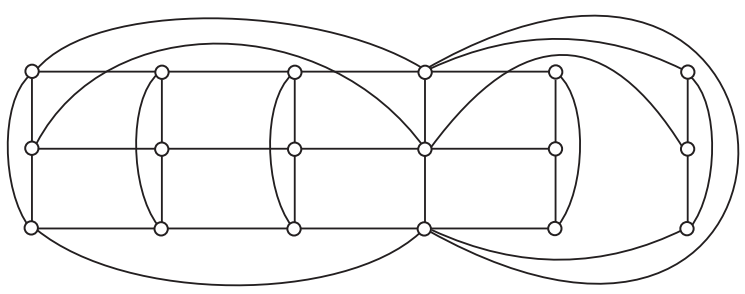

Fig. 4 The drawing of the graph $G \square C_{3}$

Assume that there is a good drawing of $G \square C_{3}$ with at most 4 crossings and let $D$ be such a drawing. The subgraph $C_{3}^{c} \cup T^{d} \cup T^{e} \cup X^{f}$ of the graph $G \square C_{3}$ is isomorphic to the graph $C_{4} \square C_{3}$ and $\operatorname{cr}\left(C_{4} \square C_{3}\right)=4$ (see [19]). Thus, in $D$ there is no crossing on the edges of $T^{a} \cup T^{b}$. The planar subdrawing of $T^{a} \cup T^{b}$ induced by $D$ is unique within isomorphism and divides the plane into two triangular and three hexagonal regions in such a way that there is no region with all three vertices $c_{0}, c_{1}$, and $c_{2}$ on its boundary. So, an edge of $T^{d}$ crosses in $D$ an edge of $T^{a} \cup T^{b}$, which contradicts the assumption that no edge of $T^{a} \cup T^{b}$ is crossed.

Theorem 3.2. $\operatorname{cr}\left(G \square C_{4}\right)=10$.

Proof. In Fig. 5 there is a good drawing of $G \square C_{4}$ with ten crossings, thus $\operatorname{cr}\left(G \square C_{3}\right) \leq 10$.

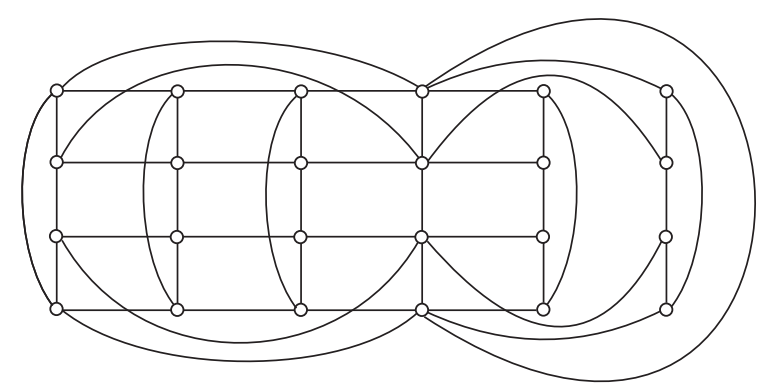

Fig. 5 The drawing of the graph $G \square C_{4}$

Assume that there is a good drawing of $G \square C_{4}$ with at most 9 crossings and let $D$ be such a drawing. The graph $G \square C_{4}$ contains the subgraph $C_{4}^{c} \cup T^{d} \cup T^{e} \cup X^{f}$ which is isomorphic to the graph $C_{4} \square C_{4}$ and $\operatorname{cr}\left(C_{4} \square C_{4}\right)=8$ (see [4]). Thus, in $D$ there is at most one crossing on the edges of $T^{a} \cup T^{b}$. Consider the subgraph $T^{a} \cup T^{b}$ of the graph $G \square C_{4}$ and let $D^{\prime}$ be its subdrawing induced by $D$.

First, suppose that $\operatorname{cr}_{D}\left(T^{a} \cup T^{b}\right)=0$. As $T^{a} \cup T^{b}$ is subdivision of the planar graph $P_{1} \square C_{4}$, the planar subdrawing of $T^{a} \cup T^{b}$ induced by $D$ is unique within isomorphism and divides the plane into two quadrangular and four hexagonal regions in such a way that there are at most two of the vertices $c_{0}, c_{1}, c_{2}$, and $c_{3}$ on the boundary of every region. 
So, in $D$, the edges of $T^{d}$ cross the edges of $T^{a} \cup T^{b}$ at least twice and it contradicts our assumption.

Next, let $\operatorname{cr}_{D}\left(T^{a} \cup T^{b}\right)=1$. The subgraph $T^{a} \cup T^{b}$ is obtained from $C_{4} \square P_{1}$ by an elementary subdivision of every edge joining two 4-cycles $C_{4}^{a}$ and $C_{4}^{b}$ and for the graph $C_{4} \square P_{1}$ there is no good drawing with exactly one crossing, because for any two edges which cross each other one can find two vertex-disjoint cycles such that crossed edges are in different cycles. Therefore two vertex-disjoint cycles cannot cross only once, the only one crossing in $D^{\prime}$ is between an edge incident with a vertex of degree two and an edge of the cycle $C_{4}^{a}$ or the cycle $C_{4}^{b}$. In this case, the cycle $C_{4}^{a}$ or the cycle $C_{4}^{b}$ separates in $D$ some vertex $c_{i}$ of the cycle $C_{4}^{c}$ from the other vertices of $C_{4}^{c}$. Hence, $C_{4}^{c}$ crosses in $D$ the edges of $T^{a} \cup T^{b}$ at least twice and this contradiction completes the proof.

Theorem 3.3. $\operatorname{cr}\left(G \square C_{5}\right)=14$.

Proof. In the drawing of the graph $G \square C_{5}$ in Fig. 6 one can easily see that $\operatorname{cr}\left(G \square C_{5}\right) \leq 14$.

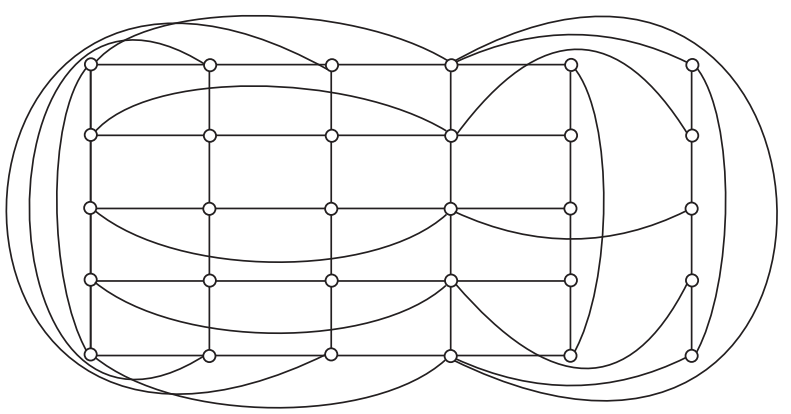

Fig. 6 The drawing of the graph $G \square C_{5}$

Assume that there is a good drawing of the graph $G \square C_{5}$ with at most 13 crossings and let $D$ be such a drawing. The graph $G \square C_{5}$ contains the graph $C_{4} \square C_{5}$ as a subgraph and $\operatorname{cr}\left(C_{4} \square C_{5}\right)=10$ (see [2]). Thus, in $D$ there are at most three crossings on the edges of $T^{a} \cup T^{b}$. Consider the subgraph $T^{a} \cup T^{b}$ of the graph $G \square C_{5}$ and let $D^{\prime}$ be its subdrawing induced by $D$.

First, assume that $\operatorname{cr}_{D}\left(T^{a} \cup T^{b}\right)=0$. As $T^{a} \cup T^{b}$ is a subdivision of the planar graph $P_{1} \square C_{5}$, the subdrawing $D^{\prime}$ of $T^{a} \cup T^{b}$ induced by $D$ divides the plane into two regions without vertices of $C_{5}^{c}$ on their boundaries and into five regions having two vertices of $C_{5}^{c}$ on the boundary of every region. If, in $D$, the cycle $C_{5}^{d}$ is placed in a region of $D^{\prime}$ with fewer than two vertices of $C_{5}^{c}$ on its boundary, then $\operatorname{cr}_{D}\left(T^{a} \cup T^{b}, T^{d}\right) \geq 5$. If $C_{5}^{d}$ is placed in a region with two vertices of $C_{5}^{c}$ on the boundary, then one vertex of $C_{5}^{c}$ is separated from $C_{5}^{d}$ by at least two vertex-disjoint cycles. Hence, $\operatorname{cr}_{D}\left(T^{a} \cup T^{b}, T^{d}\right) \geq 4$. If the cycle $C_{5}^{d}$ crosses the edges of $T^{a} \cup T^{b}$ two or three times, then it is placed in two regions of $D^{\prime}$ with at most three vertices of $C_{5}^{c}$ on their boundaries and, in $D$, the edges of $T^{d}$ cross the edges of $T^{a} \cup T^{b}$ at least four times. If there are four vertices of $C_{5}^{c}$ on the boundaries of the regions in $D^{\prime}$ in which $C_{5}^{d}$ is placed in $D$, the edges of $C_{5}^{d}$ cross the edges of $T^{a} \cup T^{b}$ at least four times.
In case 2, assume that $c r_{D}\left(T^{a} \cup T^{b}\right)=1$. As the subgraph $T^{a} \cup T^{b}$ is obtained from $P_{1} \square C_{5}$ by elementary subdivision of every edge joining two 5-cycles $C_{5}^{a}$ and $C_{5}^{b}$, and therefore for the graph $C_{5} \square P_{1}$ there is no good drawing with exactly one crossing (because for any two edges which cross each other one can find two vertex-disjoint cycles such that crossed edges are in different cycles and two vertex-disjoint cycles cannot cross only once), the only one crossing in $D^{\prime}$ is between an edge incident with a vertex of degree two and an edge of the cycle $C_{5}^{a}$ or the cycle $C_{5}^{b}$. In this case, the cycle $C_{5}^{a}$ or the cycle $C_{5}^{b}$ separates in $D$ some vertex $c_{i}$ of the cycle $C_{5}^{c}$ from the other vertices of $C_{5}^{c}$. Hence, $C_{5}^{c}$ crosses in $D$ the edges of $T^{a} \cup T^{b}$ at least twice. The removing of the separated vertex $c_{i}$ of the cycle $C_{5}^{c}$ from $D^{\prime}$ we have the drawing without crossings. This drawing divides the plane in such a way that there are at most two vertices of $C_{5}^{c}$ on the boundary of every region. As the vertex $c_{i}$ is in $D^{\prime}$ separated from the other vertices of $C_{5}^{c}$, in the subdrawing $D^{\prime}$ of $T^{a} \cup T^{b}$ with one crossings there are at most two vertices of $C_{5}^{c}$ on the boundary of a region. If the cycle $C_{5}^{d}$ of $T^{d}$ crosses the 2-connected subgraph $T^{a} \cup T^{b}$, it crosses $T^{a} \cup T^{b}$ at least two times. Otherwise $C_{5}^{d}$ is in $D$ placed in one region in the view of the subdrawing of $T^{a} \cup T^{b}$ and at least two edges of $T^{d}$ joining $C_{5}^{d}$ with the vertices of $C_{5}^{c}$ cross the edges of $T^{a} \cup T^{b}$. So, in this case, again, there are more than three crossings on the edges of $T^{a} \cup T^{b}$. It is a contradiction.

In case 3, assume that $c r_{D}\left(T^{a} \cup T^{b}\right) \geq 2$. Then at least one subgraph $T^{d}$ or $T^{e}$ does not cross in $D$ the edges of $T^{a} \cup T^{b}$. Without loss of generality, let $T^{d}$ does not cross the edges of $T^{a} \cup T^{b}$. So, $c r_{D}\left(T^{a} \cup T^{b}, T^{d}\right)=0$. In this case, $c r_{D}\left(T^{a}, T^{d}\right)=0$ and $c r_{D}\left(T^{b}, T^{d}\right)=0$. As $T^{a} \cup T^{d}$ is a subdivision of the planar graph $P_{1} \square C_{5}$, the subdrawing $D^{\prime \prime}$ of $T^{a} \cup T^{d}$ divides the plane into several regions without vertices of $C_{5}^{c}$ on their boundaries and into regions, which have exactly two vertices of $C_{5}^{c}$ on the boundary of one region. Fig. 7 shows the drawing $D^{\prime \prime}$ in which possible crossings among the edges of $T^{a}$ are inside the left disc bounded by the dotted cycle and possible crossings among the edges of $T^{d}$ are inside the right disc bounded by the dotted cycle.

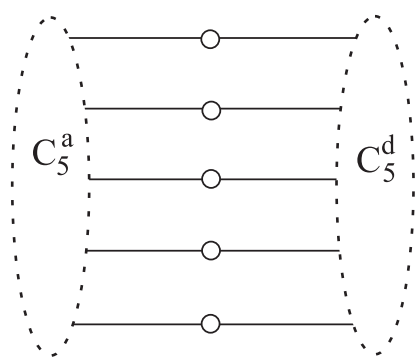

Fig. 7 The subdrawing of the subgraph $T^{a} \cup T^{d}$

We can suppose that if, in $D$, an edge not incident with a vertex of $C_{5}^{a}$ or $C_{5}^{d}$ passes through one of these two discs, then it crosses the edges of $T^{a} \cup T^{d}$ at least twice. Consider now a subgraph $T^{b}$. Both $C_{5}^{b}$ and $T^{a} \cup T^{d}$ are 2-connected graphs and so, $\operatorname{cr}_{D}\left(C_{5}^{b}, T^{a} \cup T^{d}\right) \neq 1$. If, in $D$, the cycle $C_{5}^{b}$ is placed in a region of $D^{\prime \prime}$ with fewer than two vertices of $C_{5}^{c}$ on its boundary, then $\operatorname{cr}_{D}\left(T^{a} \cup T^{d}, T^{b}\right) \geq 4$. If $C_{5}^{b}$ is 
placed in a region with two vertices of $C_{5}^{c}$ on the boundary, then one vertex of $C_{5}^{c}$ is separated from $C_{5}^{b}$ by at least two vertex-disjoint cycles. Hence, $\operatorname{cr}_{D}\left(T^{a} \cup T^{d}, T^{b}\right) \geq 4$. If the cycle $C_{5}^{b}$ crosses the edges of $T^{a} \cup T^{d}$ two or three times, then it is placed in two regions of $D^{\prime \prime}$ with at most three vertices of $C_{5}^{c}$ on their boundaries and the in $D$ edges joining $C_{5}^{b}$ with $C_{5}^{c}$ cross the edges of $T^{a} \cup T^{b}$ at least four times. If there are four vertices of $C_{5}^{c}$ on the boundaries of the regions in $D^{\prime \prime}$ in which $C_{5}^{b}$ is placed in $D$, at least four crossings between the edges of $C_{5}^{b}$ and the edges of $T^{a} \cup T^{d}$ are necessary. As $c r_{D}\left(T^{d}, T^{b}\right)=0$, all considered crossings are between the edges of $T^{a}$ and the edges of $T^{b}$. This contradiction with the assumption that there are at most three crossings on the edges of $T^{a} \cup T^{b}$ completes the proof.

\section{DISCUSSION/CONCLUSIONS}

There are open problems to detemine the crossing numbers of graphs $S_{5} \square C_{n}$ for $n \geq 5$ and of graphs $G \square C_{n}$ for $n \geq 6$.

\section{ACKNOWLEDGEMENT}

The research was supported by the Slovak VEGA grant No. 1/0309/11.

\section{REFERENCES}

[1] ANDERSON, M. - RICHTER, R. B. - RODNEY, P.: The crossing number of $C_{6} \times C_{6}$, Congr. Numerantium 118, No. 1 (1996) 97-107.

[2] BEINEKE, L. W. - RINGEISEN, R. D.: On the crossing numbers of products of cycles and graphs of order four, J. Graph Theory 4, No. 2 (1980) 145-155.

[3] BOKAL, D.: On the crossing number of Cartesian products with paths, J. of Comb. Theory 97, No. 3 (2007) 381-384.

[4] DEAN, A. M. - RICHTER, R. B.: The crossing number of $C_{4} \times C_{4}$, J. Graph Theory 19, No. 1 (1995) 125129.

[5] DRAŽENSKÁ, E.: The crossing number of $G \square C_{n}$ for the graph $G$ on six vertices, Mathematica Slovaca $\mathbf{6 1}$, No. 5 (2011) 1-12.

[6] DRAŽENSKÁ, E. - KLEŠČ, M.: The crossing numbers of products of cycles with 6-vertex trees, Tatra Mt. Math. Publ. 36, No. 2 (2007) 109-119.

[7] DRAŽENSKÁ, E. - KLEŠČ, M.: On the crossing numbers of $G \square C_{n}$ for graphs $G$ on six vertices, Discussiones Mathematicae Graph Theory 31, No. 2 (2011) 239-252.
[8] GLEBSKY, L. Y. - SALAZAR, G.: The crossing number of $C_{m} \times C_{n}$ is as conjectured for $n \geq m(m+1)$, J. Graph Theory 47, No. 1 (2004) 53-72.

[9] HARARY, F. - KAINEN, P. C. - SCHWENK, A. J.: Toroidal graphs with arbitrarily high crossing numbers, Nanta Math. 6, No. 1 (1973) 58-67.

[10] JENDROL'S. - ŠČERBOVÁ, M.: On the crossing numbers of $S_{m} \times P_{n}$ and $S_{m} \times C_{n}$, Časopis pro pěstování matematiky 107, No. 3 (1982) 225-230.

[11] KLEŠČ, M.: On the crossing numbers of Cartesian products of stars and paths or cycles, Mathematica Slovaca 41, No. 1 (1991) 113-120.

[12] KLEŠČ, M.: The crossing numbers of Cartesian products of paths with 5-vertex graphs, Discrete Mathematics 233, No. 1-3 (2001) 353-359.

[13] KLEŠČ, M.: The crossing number of $K_{2,3} \times C_{3}$, Discrete Mathematics 251, No. 1-3 (2002) 109-117.

[14] KLEŠČ, M.: Some crossing numbers of products of cycles, Discussiones Mathematicae Graph Theory $\mathbf{2 5}$, No. 1-2 (2005) 197-210.

[15] KLEŠČ, M. - RICHTER, R. B. - STOLBERT, I.: The crossing number of $C_{5} \times C_{n}$, J. Graph Theory 22, No. 3 (1996) 239-243.

[16] KLEŠČ, M. - KOCÚROVÁ, A.: The crossing number of products of 5-vertex graphs with cycles, Discrete Mathematics 307, No. 11-12 (2007) 1395-1403.

[17] RICHTER, R. B. - THOMASSEN, C.: Intersection of curve systems and the crossing number of $C_{5} \times C_{5}$, Discrete Comp. Geom. 13, No. 2 (1995) 149-159.

[18] RICHTER, R. B. - SALAZAR, G.: The crossing number of $C_{6} \times C_{n}$, Australasian Journal of Combinatorics 23, No. 1 (2001) 135-144.

[19] RINGEISEN, R. D. - BEINEKE, L. W.: The crossing number of $C_{3} \times C_{n}$, J. Combinatorial Theory $\mathbf{2 4}$, No. 2 (1978) 134-136.

Received July 23, 2012, accepted September 26, 2012

\section{BIOGRAPHY}

Emília Draženská was born on 28. 01. 1970. In 1993 she graduated Faculty of Science at Pavol Jozef Šafárik University in Košice. At the same university in 2009 she defended her $\mathrm{PhD}$ in the field of discrete mathematics. Her thesis title was "The crossing numbers of Cartesian products of graphs". Since 1994 she is working at Department of Mathematics and Theoretical Informatics, Faculty of Electrical Engineering and Informatics, Technical University of Košice. 